\title{
Automated Dynamic Alerts from Intensive Care Unit To Smart Phone using GPRS
}

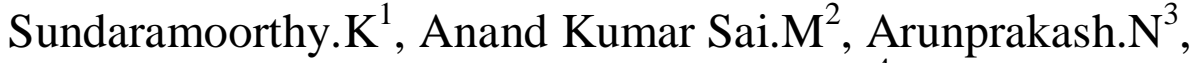 \\ Dr.S.Srinivasa Rao Madhane ${ }^{4}$ \\ ${ }^{1}$ Research Scholar, St.Peters University \\ ${ }^{23}$ Agni College of Technology \\ ${ }^{4}$ Adhiparasakthi College of Engineering, Kalavai
}

\begin{abstract}
An automated context aware dynamic alert system from an Intensive Care Unit to smart phones using GPRS was developed. The developed system collectively looks at all the available data on a patient before generating an alert. In order to achieve this, the system continuously monitors all the available vital signals before concluding the seriousness of the situation and generates an alert based on the conclusion and also a set of contexts. Setting up dynamic threshold values for vital signs based on the patient history and the current condition is another major challenge in such a system. An Internet interface allowed data inspection from a remote location and individual patient details for alert generation to be programmed through a web page. The smart alert generation system delivers the alert based on the current context of the recipient. This is made possible by continuously evaluating the various contexts of the recipients. We are implementing a smart phone based system for automation of the workflow in the ICU. This system can be made ubiquitous in the future. This paper presents the working of the smart alert system which is under development.
\end{abstract}

Index Terms: - Automation, cellular networks, Internet, Critical Care, Smart Alerts, Device interfacing, Decision Support, Hospital Information Management Systems (HIMS).

\section{INTRODUCTION}

One of the traditional roles of nurses in ICU involved surveillance. It involved constant monitoring of patient vitals like temperature, pulse, blood pressure, respiratory rate and oxygen saturation and recognizing clinical deterioration. Prompt reporting of the changes in these vital signs are very much essential and any such delays in this can affect the patient's condition. Currently monitoring in an ICU is performed on a routine basis by instruments like dual parameter monitor, multi-parameter monitor, central monitoring stations, etc. These instruments are used for monitoring the various vital parameters. The display is in the form of strip charts, alphanumeric displays and graphs. The duty doctor can set the upper and lower threshold values for different vital signs and if these limits are violated, alarms are generated in different forms based on the context. Since the limits are fixed and moreover the patient in an ICU is usually in a critical situation, violations occur regularly. Owing to this, both the duty doctor and the nurse tend to ignore these alarms.

Automated monitoring [1] of the vital parameters on a continuous basis by a real-time computing system provides the possibility of detection of unacceptable variations not only from the fixed hard limits as above but also from the near-time trends. Such an analysis can take into consideration various other factors like food intake, drug delivery, onset of fever, cough, etc., and account for expected variations due to these before deciding that the signal variation is outside limits and an alert is to be generated.[2]

Generation of an alert to various hierarchies of the clinical staff involved in the treatment of the patient needs careful planning. At the lowest level we have the duty nurses who are to be alerted about the violation. Based on the seriousness of the alert, it has to be propagated to the residents, junior doctors, next senior level doctor and finally the unit in charge. The normal practice is to leave the decision for propagation to a higher level to the person responsible at the lower level. This may lead to human errors. Thus, with automated alarm generation much better schemes of propagation is possible. The system can be programmed to dynamically compute the seriousness of the alarm condition on a continuous basis and based on this, alert the appropriate levels.

The method of delivery of the alert is another important aspect. For this, the system needs to be aware of not only the seriousness of the alert, but also the context of the recipient. An audio alert is inappropriate in case the doctor is already travelling to the ICU to attend the case. A visual alert is inappropriate to a doctor who is presently asleep. Generating audio alerts for moderate violations may be quite appropriate during day time, but is inappropriate in the late hours of the night. Thus the alert system should consider a combination of factors 
for the generation and propagation of alerts. We are developing a smart alert generation system based on continuous monitoring of the vital parameters.

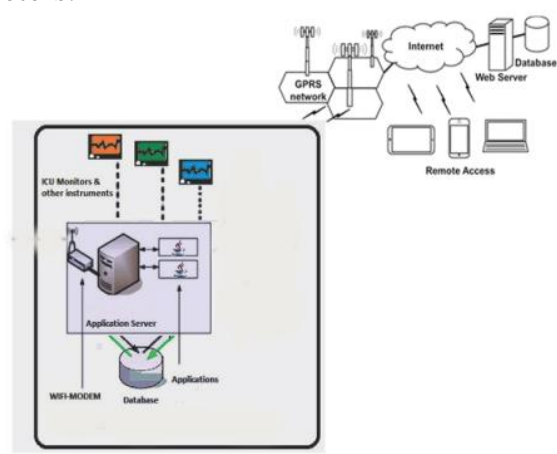

Fig.1: Architecture of ICU Automation system

\section{HOSPITAL MANAGEMENT AND ICU AUTOMATION SYSTEM}

Figure 1 gives the overall architecture of the ICU automation system. It consists of an ICU server connected to the central server using the hospital LAN. A second network card in the ICU server is used to form a Wi-Fi network to connect to the smart phone. These devices provide the primary interface for the nurses, doctors and other staff in the ICU. The ICU server has its own database and application servers deployed in it. In our implementation MySQL serves as the DBMS engine and Apache Tomcat provides the application server functionality. Hibernate provides the DBMS connectivity and Struts framework is used for application development at server side. The entire application is Web-enabled, requiring only a standard browser at the client side for the client functionality. The client side view was developed using responsive web design which allows the system to be accessed by any device and the layout automatically adjusts itself to the resolution of the display that is being used. This application allows access to the system from a remote location. This will help the doctor to be aware of the condition of the patient even when he is not in the hospital. All the clinical information is stored in the ICU server and is available to the different members of the clinical team based on their access privileges. Another advantage of the system is that it can directly read from equipment connected to the patient.

In the previous architecture they have used a WIFI connected ICU space [3] where the physicians and nurses will be provided with a Tablet PC which can be used for data entry into the system. This allows faster, accurate and easier entry of data [4] [5] into the system. Figure 2 shows a patient registration form that has to be filled before a patient is admitted in the ICU. Once the data is entered properly the different faceted views can be provided to the end users (doctors, clinical staffs and others). Thus the end users can analyze the condition of the patient within few clicks by reviewing his EMR (Electronic Medical Record) information.

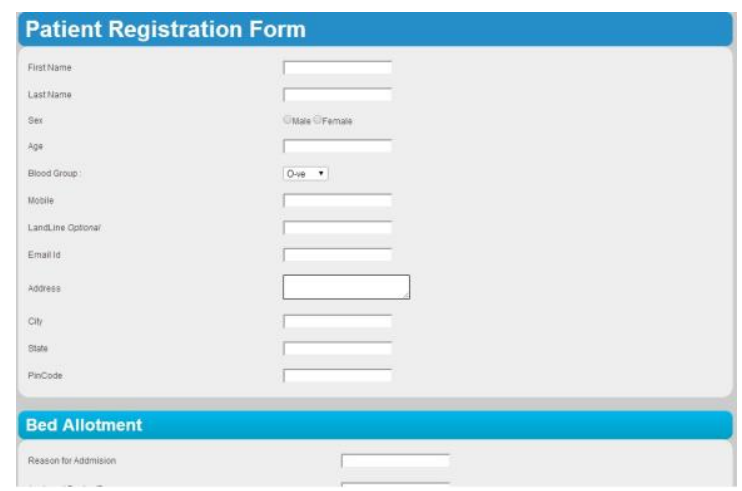

Fig.2: Patient medical record entry form

\section{PATIENT HISTORY AND LABORATORY MANAGEMENT}

In an ICU, many systems are used for collecting different data [6] [7]. These include readings from various conventional medical instruments like blood pressure measuring devices, analogue thermometers etc. Other information like time and dosage of administration of medicines, food, etc., are entered by the duty nurse. These are also significant in the alert generation analysis. 
Another important data set to be considered by the alert generator are the results from the laboratory. In our system, the lab results are captured by the PHLM (Patient History and Lab Management) server.

The following steps are involved in this procedure.

- Issuing a lab request: This is done by the duty nurses using a Tablet PC or a smart phone by invoking the Lab request activity in our application. Figure 3 shows a window in which a doctor can issue a lab request or view an existing report.

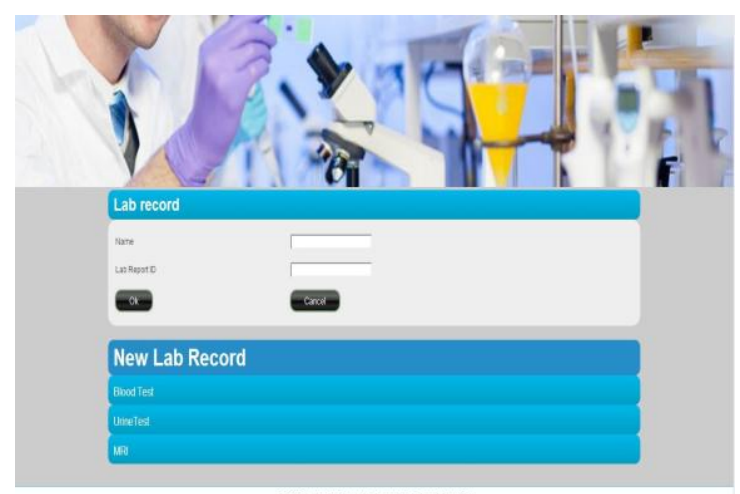

Fig.3: Issuing a lab request/ View existing lab report

- $\quad$ Result feeding: This is an activity at the laboratory. The lab assistant performs the test and feeds the results into the PHLM server. Figure 4 shows a form in which the lab assistant can enter the test results. These results can be viewed by the doctor by using the lab report ID.

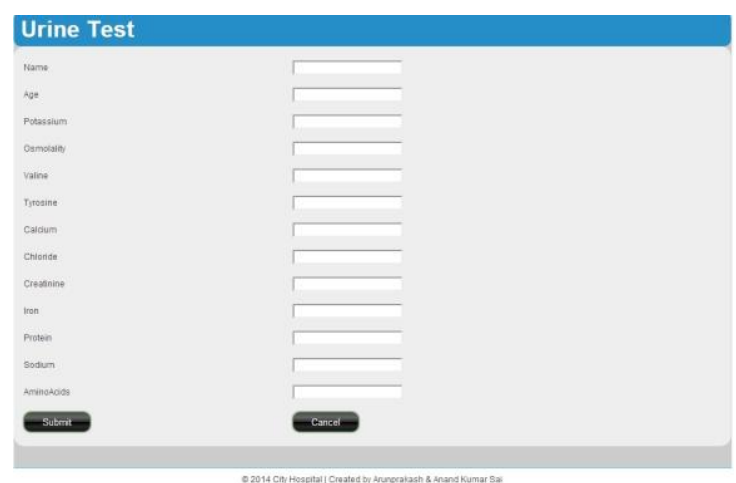

Fig.4: Lab result entry

- Viewing the results: The doctors, nurses and other clinical staff connected with ICU automation system can view the result by using the appropriate patient name and Lab Report ID. The alert system also can re-assess the criticality level based on these results. Figure 5 shows a doctor viewing a lab report.

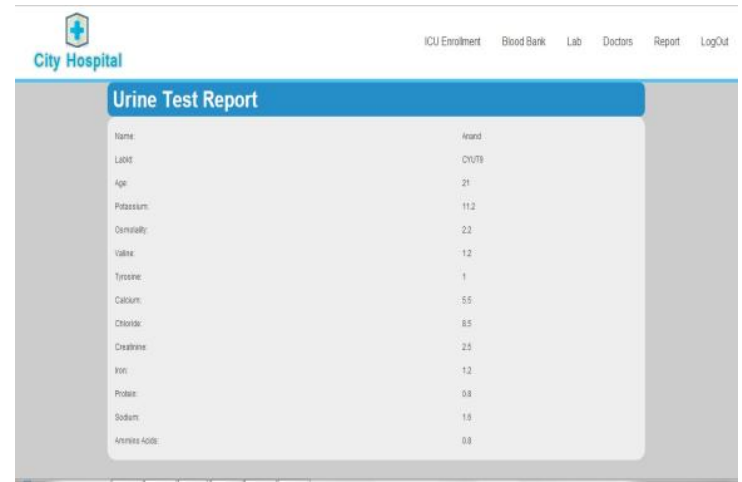

Fig.5: Viewing existing report 


\section{DYNAMIC ALERT LEVEL DETERMINATION}

The dynamic alert level determination system consists of a classifier [8] that operates on inputs from patient monitoring devices and a decision support system that operates on the output of the classifier and also the current EMR of the patient. Note that all manually generated information and the laboratory information are reflected in the EMR and hence the alert generator get all information it needs from this data structure.

The vital signs from the monitoring devices (heart rate, blood pressure, blood oxygen saturation and respiratory rate) are fed to a classifier module which can classify these signals into different labels like (Low, Normal ,High ,Very High etc.). The classifier output is fed to a decision support system which consists of a smart alarm generator and a context aware rule set. The decision support system [9] [10] considers information regarding potential drug interactions and specific patient physiological parameters.

The smart alarm generator is basically a controller module that analyze the parameter signals (corrected for its relevant side information), to identify trends like decreasing, increasing, shooting-up, dropping etc. It makes use of a rule set [11] which is a collection of rules, set up by experts in the specific medical domain. The rule set can also be customized by the chief consultant attending to the patient. An example of a representative rule is given below.

IF $<$ Pulse rate increasing $>$ AND

$<$ Systolic blood pressure is decreasing $>$

AND

$<$ Serum potassium level is increasing $>$ THEN

\{Alert[Pulse_Rate_Dropping].level++; Alert[Pulse_Rate_Dropping].advise+=Increase fluid amount\}

\section{CONTEXT AWARE ALERT DELIVERY}

Context-aware computing can be defined as "an application's ability to adapt to changing circumstances and respond according to the context of use". Any information that can be used to characterize the situation of entities (i.e. whether a person, place or object) that are considered relevant to the interaction between a user and an application, including the user and the application themselves. Context is typically the location identity and state of people, groups and computational and physical objects.

Context aware computing aims at enhancing user experience by scheduling tasks for the user based on the current context. This can result in relieving the user from performing less critical tasks or allowing to continue the current task if the criticality of the context doesn't warrant a change. The various contexts [11] relevant to the delivery of alerts and some example scenarios are described below:

\section{C1: The seriousness of the alert}

The alerts generated can be categorized into different levels using the object characteristic termed level, associated with each alarm. Based on the value of this characteristic, the alarm may be classified into five levels viz. beginning, fair, moderate, critical, and very critical. The mapping of the alarm level to alert level is dynamic and is set up by the chief consultant.

\section{C2: The current location of the recipient}

The location of the doctor is typically determined by the GPS system of the Smart Phone that he uses. It can also be determined by other programs that he uses. In the ICU automation system, any keystroke by the doctor on any of the applications provided by the system will result in re-evaluation of location of the user, from internal heuristics. Typical locations are home, hospital, moving vehicle, OPD desk, etc. The mapping of the alert delivery mechanism to the location is set up by individual recipients. These may be overridden by the chief consultant.

\section{C3: The current activity of the recipient}

The current activity of the doctor may be going for rounds, performing a surgery, sleeping etc. As in the previous case, it is generally determined by other programs that he uses. In the ICU automation system, any keystroke by the doctor on any of the applications provided by the system will result in re-evaluation of activity of the user, from internal heuristics. The mapping of the alert delivery mechanism to the activity is set up by individual recipients. These may be overridden by the chief consultant.

\section{C4: The time}

The time of the day is another parameter considered by the system for determining the mode of delivery of alert. For e.g., some alerts are to be delivered by audio alarms in night whereas text alarms may be acceptable for the same during day. 


\section{C5: The constraint specifications}

The constraint specifications provide a set of rules that indicates the delivery mode of an alert based on the recipient, his location, time and activity. These are expressed using formal code rules for each role, an example of which is shown below

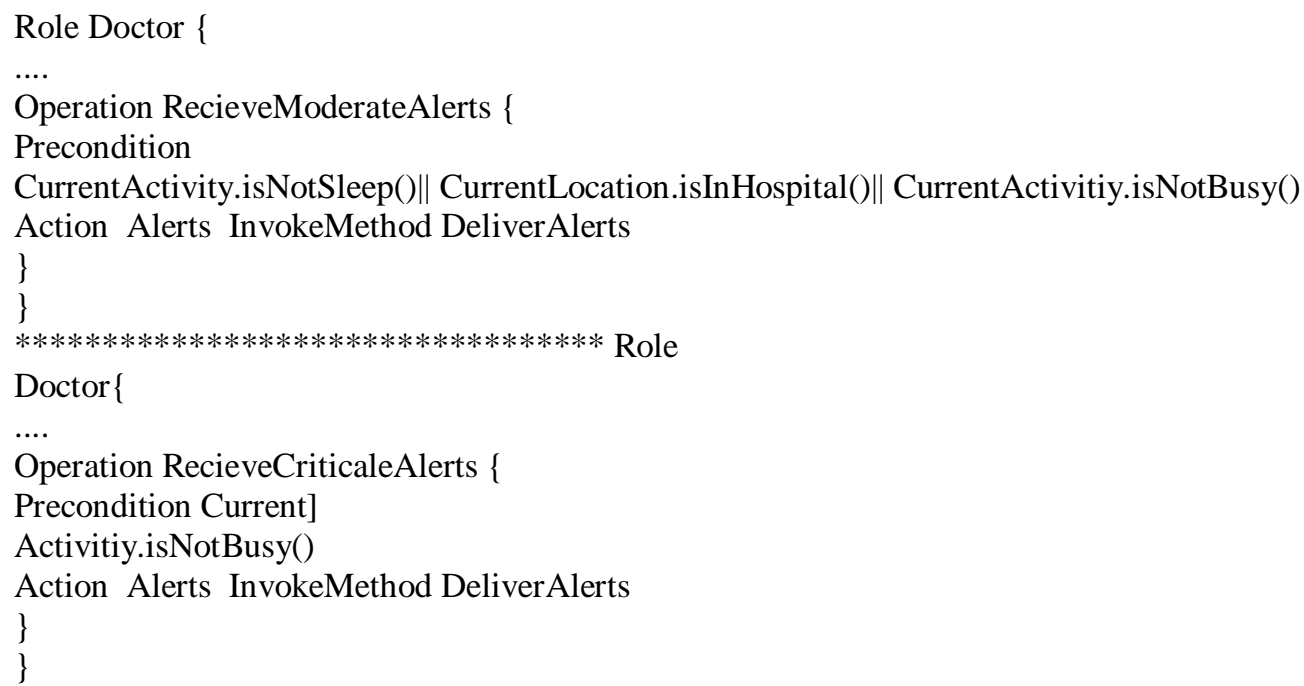

The smart alert delivery system maintains a four dimensional lattice space for each patient recipient pair. The four dimensions of this grid are alert level (0-4), location of the recipient, activity of recipient, and time of day. The value of a grid will indicate the mode of delivery of alert.

Once the Alert specifications supplied to the smart alert delivery system, it compiles this rule specification and creates an element in the corresponding grid of the patient-recipient pair. Whenever an alert is generated, it consults the corresponding grid to determine the mode of delivery.

\section{CONCLUSION AND FUTURE DEVELOPMENT}

Generation and propagation of automatic alerts from an Automated Hospital Information Management System (HIMS) will greatly enhance the effectiveness of HIMS system. At present, medical instruments tend to generate a large number of false alerts that generally gets ignored. Here we have attempted to develop a more comprehensive system that holistically looks at all available information about the situation before generating an alert. It continuously monitors all vital signals available and evaluate the cross functional effects between them before arriving at the seriousness of an alarm. The smart alert delivery system uses a mode of delivery based on the current context of the recipient based on a four dimensional grid for each patient-recipient pair, maintained by it. The chief doctor will also be able to configure and set up the system for alert generation, for each patient. The current implementation is a Tablet PC based system for automation of the workflows in an ICU. We have developed the system which allows automation of workflows using a smart phone. The system also supports GPRS network.

This system requires a more advanced security to protect Electronic Medical Records of the patients in the hospital system. Thus a security module placed at the heart of the information could be the future of the system.

\section{REFERENCES}

[1] Joaquín Gutiérrez; Juan Francisco Villa-Medina; Alejandra Nieto-Garibay; Miguel Ángel Porta-Gándara - "Automated Irrigation System Using a Wireless Sensor Network and GPRS Module. ", 2014.

[2] Joms Antony, Bichu Vijayan, Sudhin Joy, Santhosh Kumar G.S - " A Context Aware System for Generation and Propagation of Automated Alerts from an Intensive Care Unit. ", 2014.

[3] Mengling Feng; Zhtio Zhang: 1-eng Zhang; Yu Ce; Liang Yu Loy; Vellaisamy, K.; Wenyuan. Guo; Pei Loon Chin; King, N.K.K.; Beng 'Ti Ang; Cuntai Guati. - " iSyNCC: An intelligent system for patient monitoring and clinical decision support in Neuro-Critical-Care.", 2011.

[4] Khedo, K.K. ; University of Mauritius - "Context-Aware Systems for Mobile and Ubiquitous Networks.

[5] Fischer, N. ; Smolnik, S. - " The Impact of Mobile Computing on Individuals, Organizations, and Society - Synthesis of Existing Literature and Directions for Future Research.” 
[6] Bosnian RJ; Rood K; Oudemans-van Srraaten HM; Van der Spoel JI Wester JP; Zandstra DF. "Intensive care information system reduces documentation time of the nurses after cardiothoracic surgery. "

[7] Insup Lee. Oleg Sokolsky, Sanjiatn Chen, Challenges and Research Directions in Medical CyberPhysical Systems. Invited Paper in Special Issue on Cyber-Physical Systems, Proceedings of the ILLL, vol. 100, no, 1, pp,75-90. Jan, 2012.

[8] Shashi Gunawardane. Ravindra Koggalage: Ranga Rodrigo, Senata Ra-japakse, A Computer-Based ICU Patient Alert And Decision Support System, SLJOC, 2009, vol 1, 35-41.

[9] Fortier, P.; Jagannathan, S,; Michel, H,; Dluhy, N.; Oneill, K, Development of a hand-held real-time decision support aid for critical care nursing, HICSS 2003

[10] Yi Mao; Yixin Chon; Hackmann, G; Minmin Chen; Chenyang Lu: Kollef. M.; Bailey, T.C. Medical Data Mining fur Early Deterioration Warning in General Hospital Wards. ICDMW.2011; 1042-1049

[11] Nathalie Bricon-Soufa. Conrad R, Newmanb. Context awareness in health care: A review. International Journal of Medical Informatics 76 (2007)212 